\title{
Online Diagnostic Expert System for Detection of Breast Cancer in Saudi Arabia
}

\author{
Yousuf Qawqzeh, Ph.D. \\ Assistant Professor \\ Department of CSI \\ College of Science, Al- Majmaah University, KSA
}

\author{
Khalid Nazim Abdul Sattar, Ph.D. \\ Assistant Professor \\ Department of CSI \\ College of Science, Al- Majmaah University, KSA
}

\begin{abstract}
Breast ailments have affected women since the time of the pharaohs, and can be traced back to the beginning of recorded medical history. It still poses a significant threat to the population. Statistics from the National Cancer Institute place breast cancer at the top of the list of all forms of cancers afflicting women and treated there. In the Kingdom of Saudi Arabia (KSA), breast cancer constitutes $18 \%$ of all cancers in Saudi women. In our work we have proposed a prototype (website) which allows the interaction between the patient and the expert system through system's interface. Our approach includes collaborative information provisioning by the community members in additions to other sources of information like online organizations and the experts in the field [3].
\end{abstract}

\section{Keywords}

Knowledge Base (KB), Breast Cancer, Mammogram, Magnetic Resonance Imaging (MRI), Positron-Emission Tomography (PET), Fine Needle Aspiration (FNA).

\section{INTRODUCTION}

Breast cancer is a malignant tumor (a collection of cancer cells) arising from the cells of the breast. Although breast cancer predominantly occurs in women it can also affect men [3].

Breast cancer is on the rise. Big countries have the most cases but not always the highest incidences and rates in the developing world may be even higher than the official data suggest.

Ezzat et.al have discussed in their work about the complete breast cancer statistics of women in Kingdom of Saudi Arabia (KSA) in comparison with the western countries. The data collected by them as per the records from the National Registry of Cancer Tumors under the supervision of the Ministry of Health, it is found that, the infection rates is more in the eastern province when compared to the other major cities in the kingdom.

Furthermore, $70 \%$ of those infected reach late stages of cancer, reducing the chances of cure and recovery. The reason behind delay in receiving treatment and medical care is the lack of public knowledge about its symptoms, which has created an impact on the overall country resources [3].

Dr. Dalal Tamimi, an award-winning doctor who is working to build a breast cancer research center in Saudi Arabia, confirms the cultural stigma attached to the disease: "Once [women] know the diagnosis, they keep it as a secret. They don't want anybody to know it, and they don't want to talk about it." [4].

Until recently, the clinical classification of breast cancer has been based on morphologic characteristics of the tumor such as size, lymph node status, histology as well as the expression of particular bio-markers.

The high incidence for breast cancer is not unique in Middle East. Study reveals that in USA, most of the women have Breast cancer as a common ailment, which has further focused more to educate the women about early detection, and prevention [6].

Breast cancer has plenty of myths and facts surrounding its causes and risk factors. Although the exact cause has not been identified, several risk factors have been determined for this disease. Age, hereditary factors, carcinogenic elements, weight, race, age at pregnancy, smoking, alcohol consumption, abortions, and even night shift work are one of the reasons said to have caused breast cancer or increased the risk[1,6]. Certain changes in DNA can cause normal cells to turn malignant. These alterations to the DNA can be the result of one high risk factor or a combination of multiple risk factors

The main objectives of our work include awareness through education, and providing diagnostic breast care services for those in need.

We also focus to promote god breast health through breast cancer awareness, education, and research, providing diagnostic breast care service, and to support to women in all social and economic strata.

\section{LITERATURE SURVEY}

\subsection{Overview of Breast Cancer}

Breast cancer is on the rise. Big countries have the most cases but not always the highest incidences and rates in the developing world may be even higher than the official data suggest.

The simplest definition of breast cancer is the uncontrolled growth of breast cells. It is found that one out of every seven women will be diagnosed with breast cancer if all live to their full life span in a well-developed country like USA alone, [6, 10]. Ongoing focused research for breast cancer causes and cure is offering new hope for effective treatments that attack the tumor without destroying the surrounding tissue. 


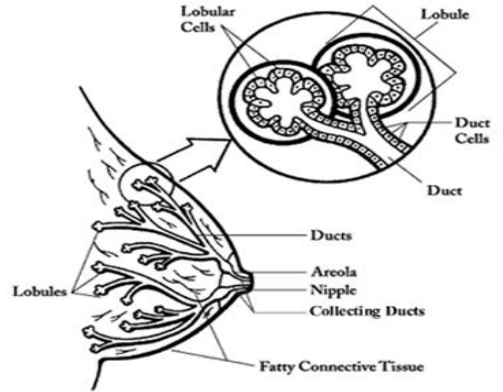

Fig. 1 : Normal Breast Structure

I. Presence of cancer refers specifically to a malignant tumor. Their characteristic properties that set them apart from benign tumors are:

II. They have no control over their growth and feed off the healthy cells

III. They metastasize i.e. cancer cells can enter the blood stream and be carried to more compatible sites in the body.

\subsubsection{Types of breast cancer}

Breast cancer can be subdivided into various classes based on the cells that are affected, the origin of the malignancy and the size and spread of the cancer. The different types of breast cancers can be listed as follows:

1. Ductal carcinoma in situ (DCIS): Most common type of non-invasive breast cancer in which only the duct walls are affected. This type of cancer does not spread through the walls of the duct to the outer tissue and hence is the most curable type of breast cancer.

2. Lobular carcinoma in situ (LCIS): The glands are affected in this particular type of cancer but the cancer is contained within the lobules and does not spread to the outer tissue.

3. Invasive ductal carcinoma (IDC): The most common form of breast cancer in which the malignancy starts in the duct and further invades the tissue from where it can be spread to the distal parts of the body.

4. Invasive lobular carcinoma (ILC): In this type the malignancy originates in the lobules or milk glands and further invades the wall to spread to the rest of the body.

5. Inflammatory breast cancer (IBC): This is a very uncommon type of invasive breast cancer in which there is no defined lump in the breast. Instead the breast skin has a thick red inflammatory look which is actually caused by cancer cells blocking lymph vessels in the skin. Instead, inflammatory breast cancer (IBC) makes the skin of the breast look red and feel warm and gives the skin a thick, pitted appearance that looks a lot like an orange peel. Pathologic changes such as cancer cells in the lymph ducts in the skin (dermal lymphatic) are characteristics of inflammatory breast cancer.

\subsubsection{Diagnosis of breast cancer}

Cancer of any type is usually asymptotic and does not show significant symptoms until it is well advanced. Hence the need to perform a routine screening test is vital in diagnosing cancer at an early stage. Breast cancer if diagnosed well within an early period of time offers high chances of survival for the patient. There are numerous diagnostic procedures and screen tests currently carried out for screening and diagnosis of breast cancer. The screen procedures include:

1. Mammogram: It is a type of imaging technique that uses a low dose x-ray system to examine the breast tissue. A mammography image is shown in Figure 2.

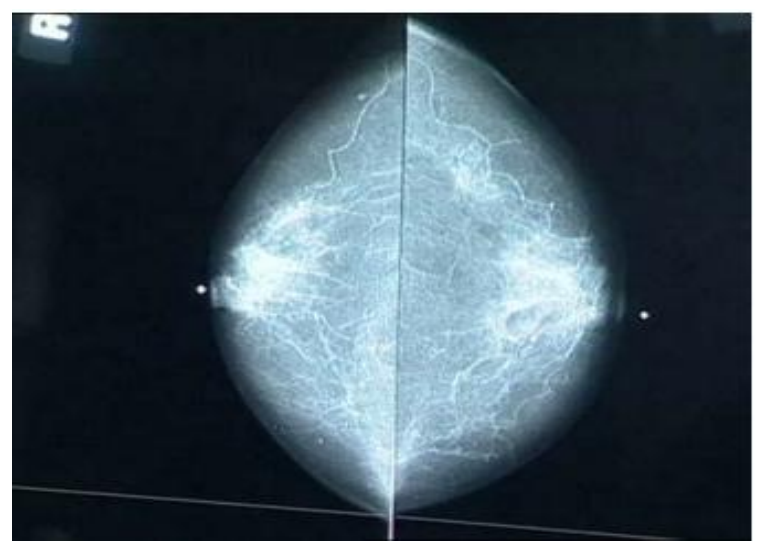

Fig. 2: A Mammography Image

Digital mammography, also called full field mammography makes use of solid state detectors that convert $\mathrm{x}$-rays into electrical signals. These electrical signals are then converted to digital images by interfacing them with a computer. Different software is then used to quantify and detect abnormal areas of density, mass or calcification as pointers for presence of malignant conditions $[5,7,8]$.

Mammograms can detect changes in the breast up to nearly two years before a physician or the patient can be aware about them. Screening mammograms comprise of a total of four views of the breast. Two standard views are taken of the right breast and two of the left. Cardio-caudal view and lateral view are the two separate views used to derive four images [8].

2. Clinical Breast Exam (CBE): A clinical breast examination is a physical examination of the breasts by a certified health professional. These examinations are used in conjunction with mammograms to detect the presence of lumps and also to check for other breast abnormalities such as mastitis or fibro adenoma [8].

3. Breast Self-Exam (BSE): In this test the individual is asked to self-examine the breast for changes in texture, appearance, weight and volume.

4. Magnetic Resonance Imaging (MRI): MRI comprises of powerful magnets that act in conjunction with radio waves to produce computer images showing differences in the number of blood vessels in various types of the tissues in the body. The radioactive dye is picked up faster by cancerous tissue than normal or benign ones. This test is used in conjunction with a mammogram for a full body screen for a complete diagnosis [9].

5. Genetic Test: In case of patients with a strong background history of ovarian and breast cancer a gene test is carried out in which the mutations if any on genes BRCA1 and BRCA2 are studied to predict the risk factor for malignancy.

6. Positron-Emission Tomography (PET): PET is similar to $\mathrm{X}$-rays where instead of cell structure the cell activity is 
shown. Cancer cells use up sugar faster than normal cells do. PET is highly accurate at diagnosing whether a tumor is cancerous.

\subsubsection{Breast cancer staging}

Breast cancer staging or grading is based on size of the tumor, whether lymph nodes are involved, whether cancer has spread beyond the breast and whether the cancer is invasive or noninvasive. The cancer stage is determined in order to best understand the prognosis and guide the treatment decisions and tailor the treatment according to the individual patient's case requirements.

There are four defined stages of breast cancer each with further subcategories [10].

1. Stage 0: Used to describe non-invasive breast cancers such as DCIS (Ductal Carcinoma In Situ) and LCIS (Lobular Carcinoma In Situ). In stage 0 there is no evidence of cancer cells or non-cancerous abnormal cells breaking out of the part of the breast in which they originated or of invading normal neighboring tissue.

2. Stage I: Describes invasive breast cancer which invades the adjacent tissue in which:

a) The tumor measures up to 2 centimeters

b) No lymph nodes are involved.

3. Stage II: This stage is divided into subcategories as IIA and IIB. II A describes invasive breast cancer in which:

a) No tumor seen in breast but seen in axillary lymph nodes

b) Tumor measures $2 \mathrm{cms}$ or less and has spread to the axillary lymph nodes.

c) Tumor larger than $2 \mathrm{cms}$ but smaller than $5 \mathrm{cms}$ and has not spread to the axillary lymph nodes.

IIB describes invasive breast cancer in which:

a) The tumor is larger than $2 \mathrm{cms}$ but not larger than $5 \mathrm{cms}$ and has spread to the axillary lymph nodes.

b) Tumor is larger than $5 \mathrm{cms}$ but has not spread to the axillary lymph nodes.

4. Stage III: This stage is subdivided into three categories IIIA, IIIB and IIIC. IIIA describes invasive breast cancer in which:

a) No tumor is found in the breast but found in axillary lymph nodes that are clumped together or sticking to other structures.

IIIB describes invasive breast cancer which:

a) The tumor may be of any size and has spread to chest wall and/or skin of the breast AND may have spread to axillary lymph nodes that are clumped together. Or it may have affected lymph nodes near the breastbone.

b) Inflammatory breast cancer is considered at least stage IIIB.

IIIC describes invasive breast cancer in which:

a) The tumor may not be in the breast or it may be of any size and has spread to chest wall or skin AND

b) The cancer has spread to lymph nodes above or below the collarbone AND

c) Cancer may have spread to axillary lymph nodes or to lymph nodes near the breastbone.
5. Stage IV: Describes invasive breast cancer in which:

The cancer has spread to other parts of the body like lungs, liver bone or brain.

\subsubsection{TNM staging system}

The TNM staging system is used by physicians to accurately define the spread of the cancer. The TNM is determined as follows:

a) $\boldsymbol{T}$ : Size of the tumor is indexed by this variable.

b) $N$ : Lymph node involvement is defined by value of $\mathrm{N}$.

c) $\boldsymbol{M}:$ whether the cancer has metastasized is denoted by $\mathrm{M}$.

\subsubsection{Breast cancer biopsy}

A final diagnosis for presence or absence of breast cancer cannot be made until a biopsy is performed and the pathologist looks at the biopsy tissue under the microscope. Only the pathologist is qualified to make the final diagnosis. The information gleaned from the gross pathology is the size, location and the character of the specimen tissue as a whole, and the size, location and character of the cancer, if present contained within it $[7,8]$.

There are two types of biopsy procedures involved. Needle biopsy, also called stereotactic biopsy and involves far less tissue. This is further subdivided as Fine Needle Aspiration (FNA) and a core needle biopsy technique.

Cytopathologists perform FNA procedure which has the advantage that it is the quickest way to demonstrate the presence of cancer. Positive diagnosis of FNA resolves the issue of presence of cancer, whereas a negative FNA gives no meaningful information. FNA is carried out with a small syringe and needle which are used to aspirate some cells from the lump for an examination under the microscope.

A core needle biopsy is performed if the lump is palpable. While the FNA procedure obtains only cells a core needle obtains a thin sample of the tissue itself and views all the cells in their proper architectural relation to other cells in the tissue.

Thus it gives more information than FNA. The second type is the open biopsy which consists of a surgical incision directly above the area to be removed and removal of some or all involved tissue. This is further subdivided into excisional biopsy or lumpectomy and incisional biopsy.

\subsubsection{Treatment options for breast cancer}

1. Surgery: Surgery is usually the first treatment option for any stage or kind of cancer. The kind of surgery depends on the size of the tumor, the spread of the cancer, the age and sex of the patient. In case the tumor is under $2 \mathrm{cms}$ and the cancer is not determined as aggressive, then a more conservative approach for surgery is approached called as lumpectomy. In this kind of surgery only the tumor and affected nodes are removed whereas the breast mass is kept intact [6].

In case of aggressive cancers usually a mastectomy is recommended by physicians, which involves complete removal of the breast.

2. Chemotherapy: Chemotherapy is the process of administering drugs through the bloodstream and affects the entire system. The treatment is used to target and eliminate the fast growing cancer cells. Unfortunately since these drugs cannot differentiate between healthy and cancer cells but eliminate only fast dividing cells, 
it also causes considerable damage to healthy cells. For example the cells which are responsible for hair growth or nails. [6]

3. Radiation Therapy: Unlike chemotherapy radiation therapy is more targeted and localized only to affected areas. The radiation treatment can be either external or internal. In internal radiation catheters are used to administer the drugs at the specific area. The type and amount of radiation is usually determined by the time of the surgery performed.

4. Hormonal Therapy: Hormonal therapy also involves the whole body and it involves medicine which is used to lower the risk of hormone receptor positive breast cancer. The hormonal treatment is based on the age of the woman and her menstruation and menopausal stages.

5. Targeted Therapies: These are highly focused treatments in which the protein that enables the rapid growth of the cancer cell is inhibited. This is less harmful to healthy cells in comparison to chemotherapy. These drugs modify the properties of cancerous cells and try and contain the growth.

Complementary and Holistic Medicine: Even though this stream is not considered to be an integral part of medical sciences, it still is an effective option for breast cancer treatment. This treatment is used in complement with surgical procedures or chemotherapy and radiation procedures. Complementary medicine involves a wide range from yoga and meditation to nutrition science and herbal medicines [2, $6]$.

\subsection{Related Works}

\subsubsection{Virtual Doctors Program}

A virtual doctor is a method of obtaining an online symptom diagnosis. With this program, the user can interact with the system by entering the primary symptoms; the program interacts with the user with a series of questions, which are designed to guide the program to ultimately provide suitable diagnosis for the same to the user.

This web site examines diseases in general, for children, women and men. A sample snapshot of the same is as shown in Figure 3 and Figure 4 below.

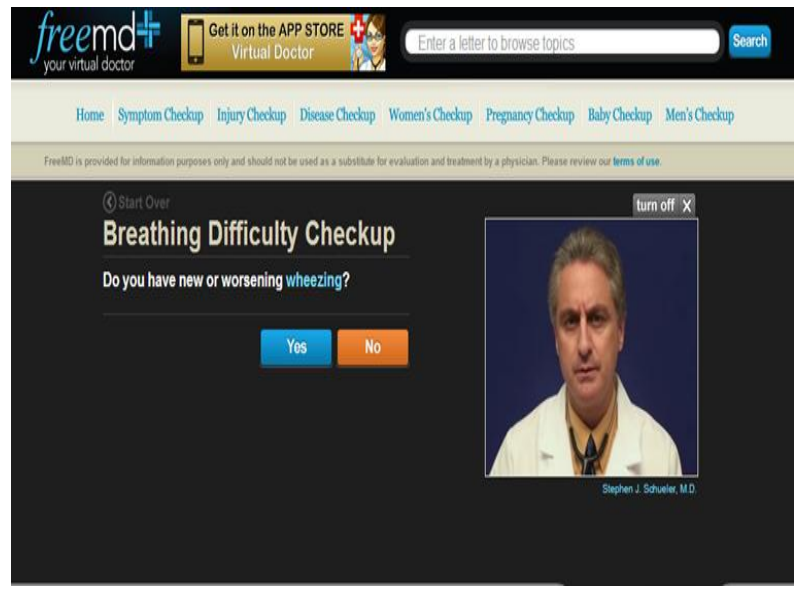

Fig. 3: Online free medical diagnostic portal

\section{Your Results}

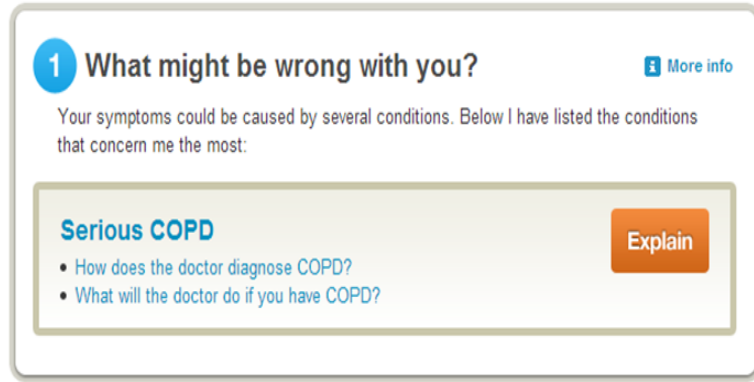

Fig. 4: Result for the query with suitable actions

\section{METHODOLOGY}

\subsubsection{Rule-based reasoning applications} architecture

An expert system is a computer system that emulates the decision-making ability of a human expert. Expert systems are designed to solve complex problems by reasoning about knowledge, like an expert, and not by following the procedure of a developer as is the case in conventional programming [14].

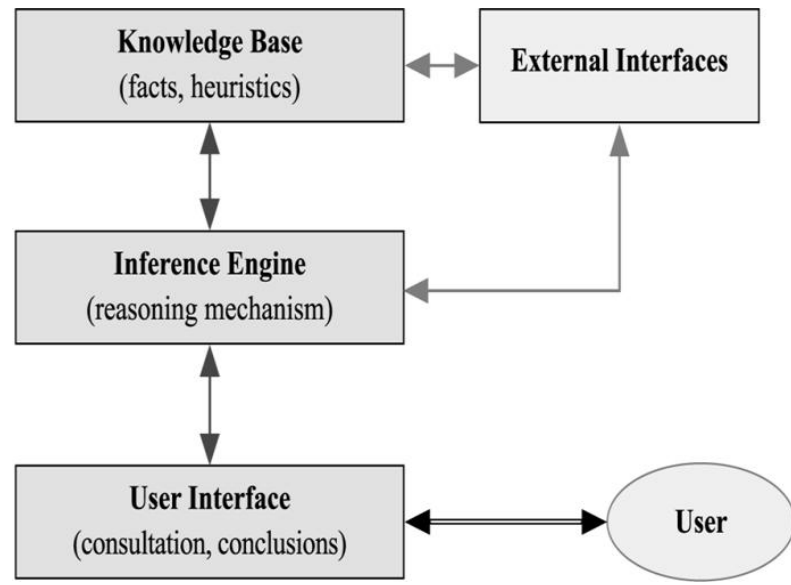

Fig. 5: Typical Rule-based Application Architecture

\subsubsection{Collaboration Diagram - Risk Assessment} Tool

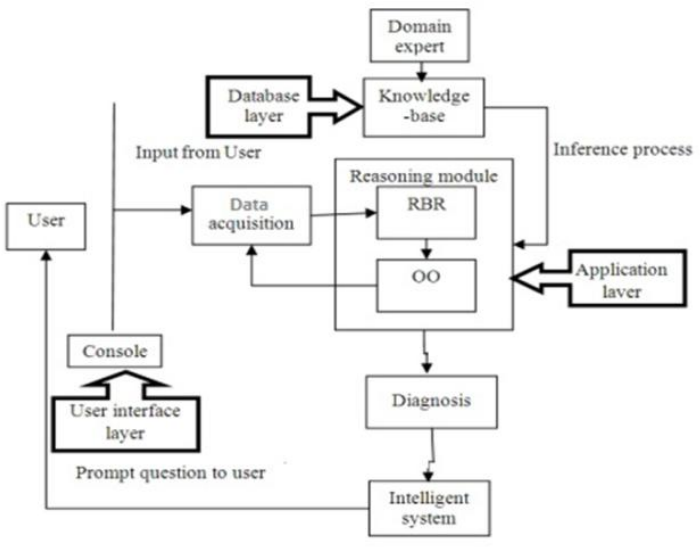

Fig. 6: Risk Assessment - Collaboration Diagram 
The main aim of our work was to develop a diagnostic tool that enables humankind in arab world to diagnose them for any abnormality in breast formation.

The following objectives were set as a benchmark while developing the application as listed below:

1. To build a culturally sensitive, web-centric support system so as to enhance the quality of life for women with breast cancer and their families.

2. Educate the public about breast cancer, the importance of early detection, and eliminate the feel of fear of having breast cancer.

3. Develop awareness about breast cancer treatments, screening and diagnosing techniques through latest technologies.

4. Facilitate access to diagnosis \& treatment centers across KSA.

5. Web application for breast cancer self-diagnosis / detection.

6. Interactive map for screening providers in the middleeast.

7. Multimedia enabled content.

8. Blog-based articles are provided.

9. FAQ section is provided.

10. Subscription-based newsletter module is provided.

A questionnaire was prepared and discussed with one of the specialist at TAIBAH medical center as stated below:

1- Is Age a key element in the diagnosis of breast cancer? Mention the typical age group advisable for the diagnosis

2- On detection of breast cancer, the treatment could be less responsive, or more responsive?

3- Should the patient visit the doctor on being diagnosed with one of the symptoms?

4- Is breast pain is a symptom of breast cancer?

5- Does every lump in the breast considered cancerous?

6- Is menstruation at an early age considered a factor for breast cancer?

7- What is the appropriate age to start selfexamination? And when mammogram is recommended?

8- What are the procedures that could be proposed before and after diagnosis?

And from the answers of these questions we extract these Rules:

1. If (different breast size) Then (nipple swells

2. If (nipple turn inward AND lump underarm) Then (nipple discharge)

3. If (lump underarm) Then (nipple discharge)
4. If (nipple discharge) Then (BC)

5. If (breast skin irritation) Then (BC)

6. If (breast pain) Then (No BC)

7. If (age>40 AND nipple swell) Then (BC)

8. If (nipple discharge OR breast skin irritation) Then (BC)

As shown in Figure 5, the typical rule based architecture was used while formulating the set of rules to develop our application.

\section{IMPLEMENTATION}

\subsubsection{Communications Interfaces}

To use the portal efficiently, the user needs to have an internet connection with a personal computer running with an Operating System like Winx or Linux in addition with a web browser and email client on his/her machine for the same.

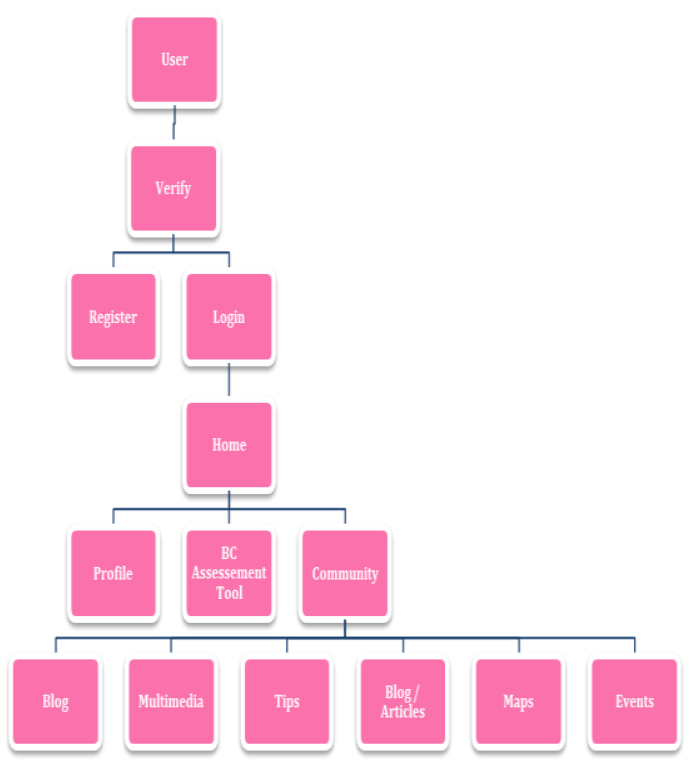

Fig. 7: Functional hierarchy

The functional hierarchy is as shown in the Figure 7 above, describes the relationships between the modules that were used to develop the web portal.

\subsubsection{Assumptions and Dependencies}

The following assumptions and dependencies were taken into consideration while developing the application and are as listed below:

I. Central server of the system must be able to handle all the incoming requests simultaneously.

II. Back up of the databases in case of hardware failure, disaster, natural calamities.

III. No data loss in case of handling of the system by the administrators or the system related personnel.

\subsubsection{Sequence Diagram}

The sequence diagram shown below in Figure 8 gives the complete description about the different modules and their interactions as used in the application. 


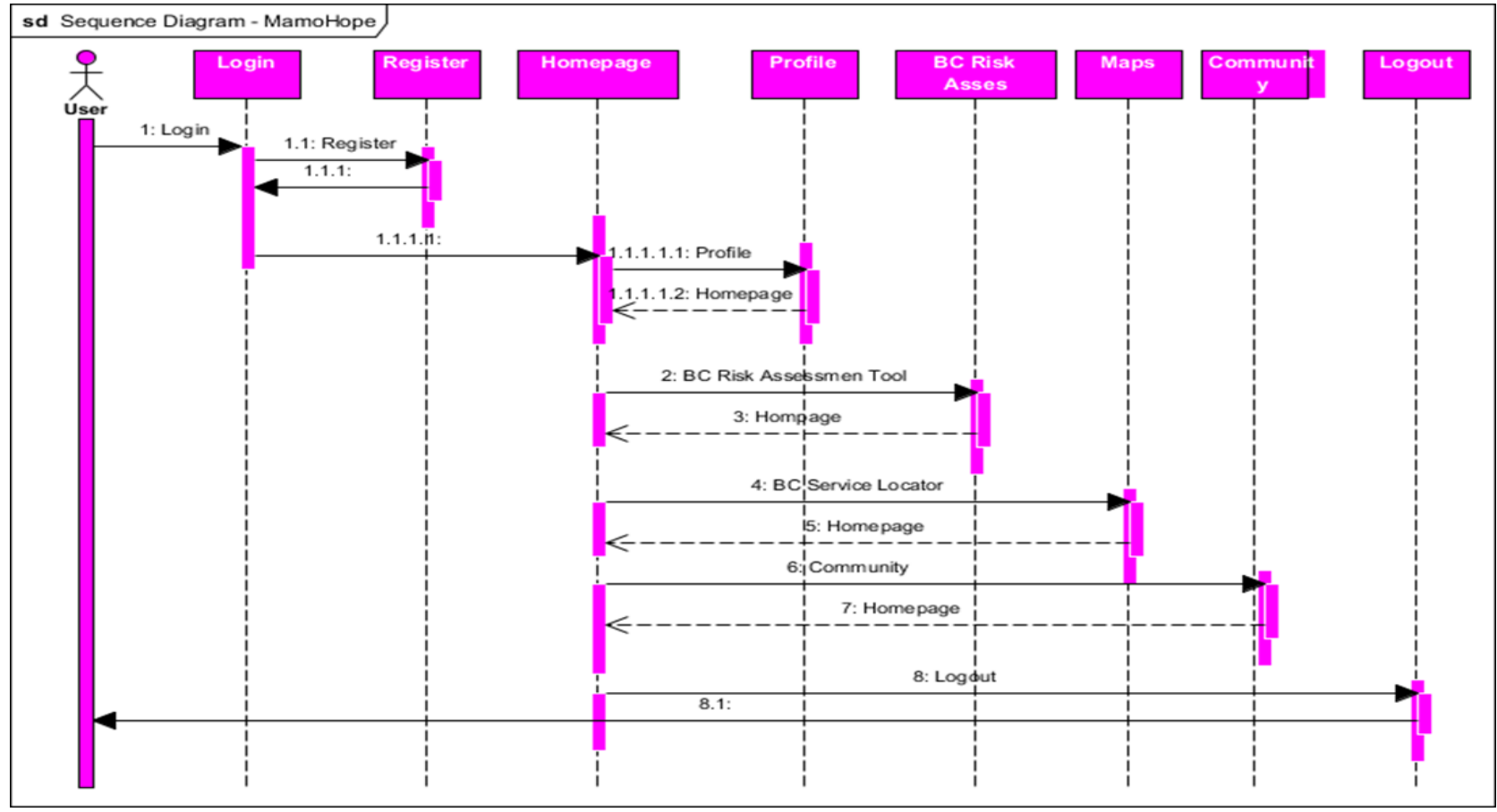

Fig. 8: MammoHope - Sequence Diagram

\section{EXPERIMENTAL RESULTS}
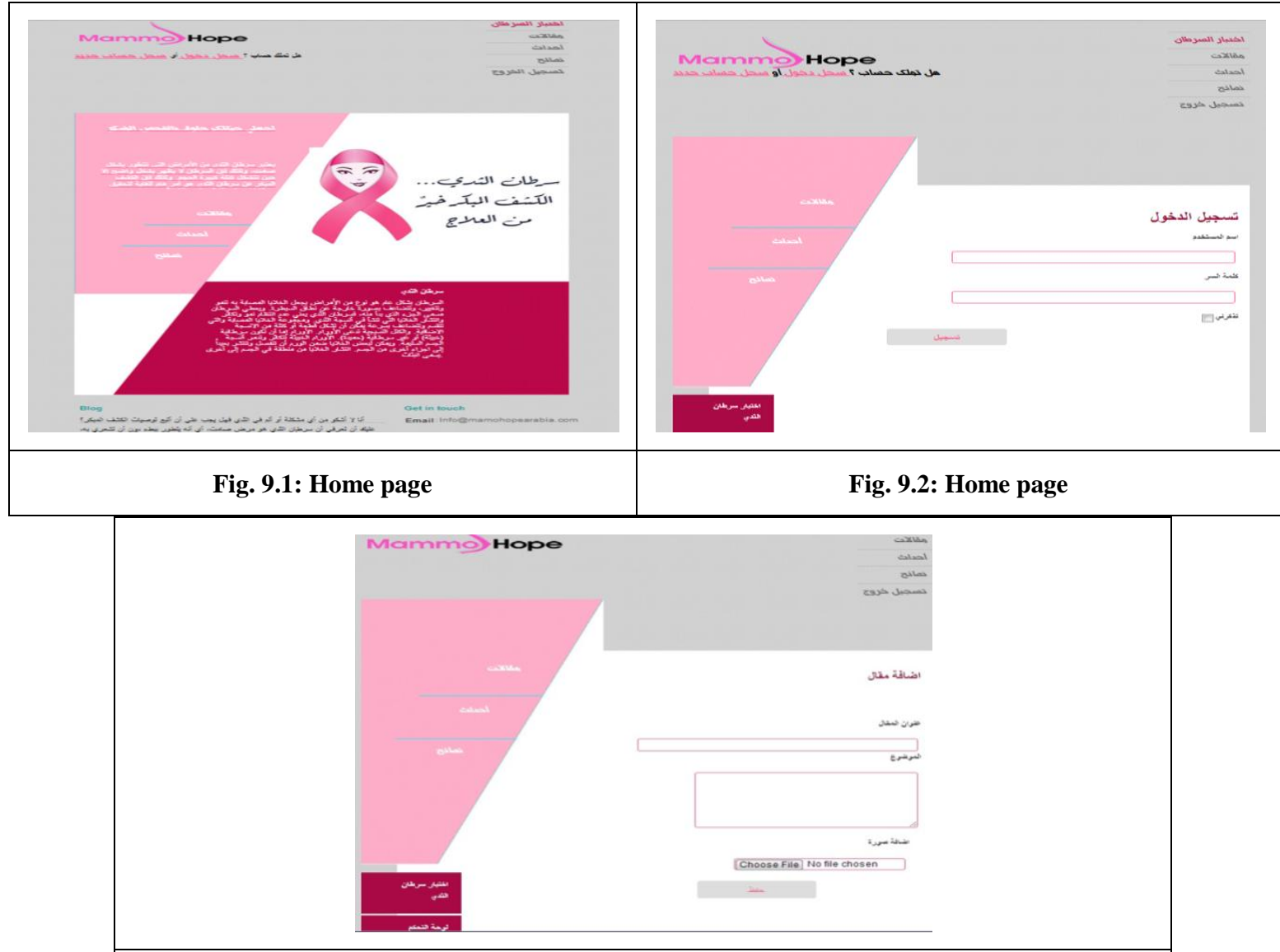

Fig. 9.3: Add article / Enter Query 


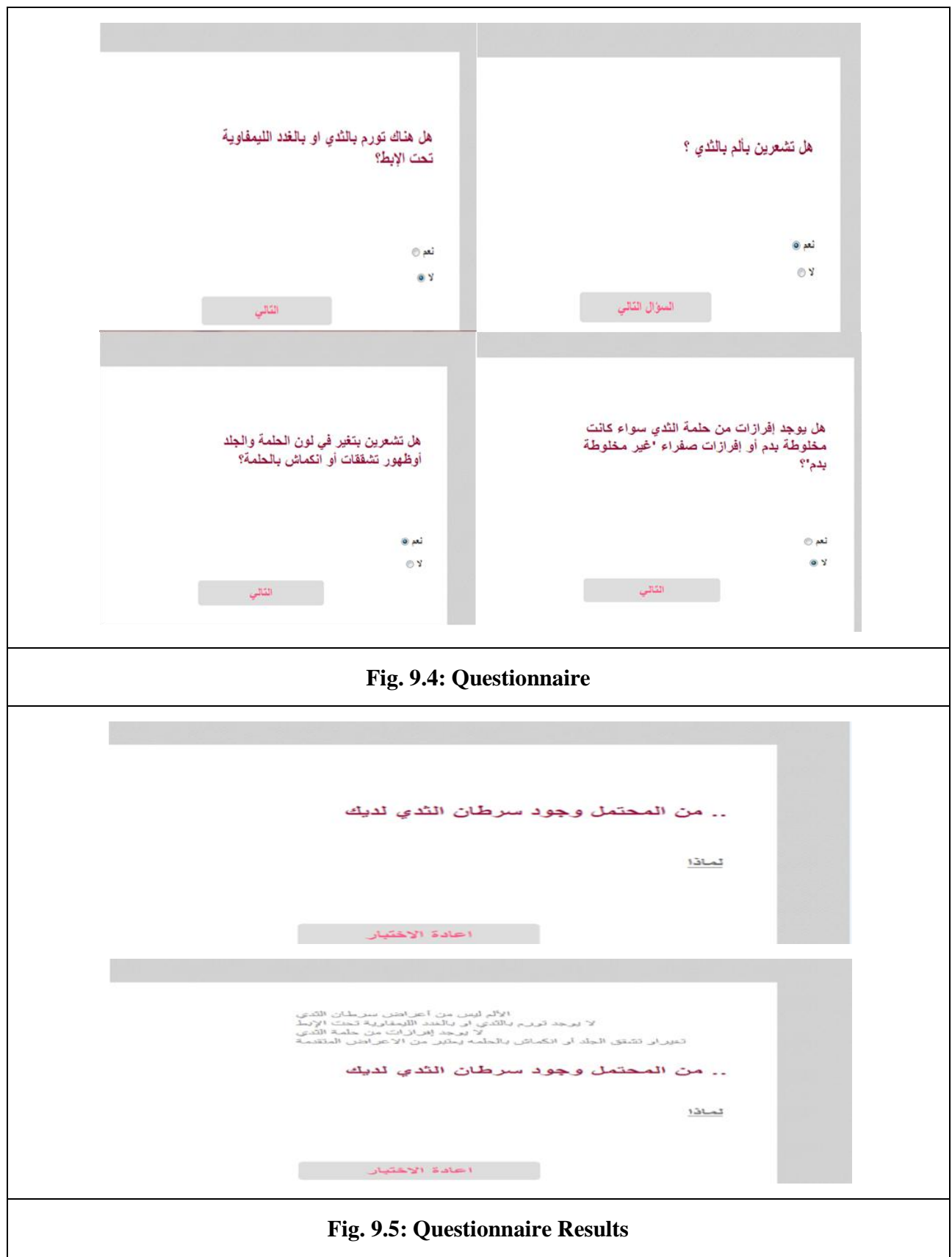

Fig. 9: Snapshots of the various user interfaces

\section{DISCUSSION AND CONCLUSIONS}

This paper aims to promote breast health through breast cancer awareness, education, and research, providing diagnostic breast care service, and to support to women in all social and economic strata. However, the developed system provides an online diagnosis tool for breast cancer anywhere and anytime for each patient from its knowledge-base. The limitation of this work is the inability to test the system in real time with breast cancer patients. There are a lot of features that could be added to the website to be a step toward serving the community better, in terms of Blogs, Chat Rooms and online interaction with experts.

\section{ACKNOWLEDGMENTS}

We would like to sincerely thank the Taibah center for breast cancer early detection, Al- Madinah City- KSA.

\section{REFERENCES}

[1] http://www.cancer.org/acs/groups/content/@nho/docume nts/document/acspc-024113.pdf.

[2] http://www.medicinenet.com/breast_cancer_facts_stages/ article.htm.

[3] M. I. Ezzeldin , F. A. Al-Mulhim and R. K. Stuart, 1998,Breast cancer in the Eastern Province of Saudi Arabia, Stockton Press, London.

[4] Ezzat AA1, Ibrahim EM, Raja MA, Al-Sobhi S, Rostom A, Stuart RK ,1999, Med Oncol; 16(2):95-103.

[5] A. P. Giuseppe Boccignone, 2000, Computer Aided Detection of Micro calcifications in Digital Mammograms, in Computers in Biology and Medicine, pp. 267-286. 
[6] Grobstein, R.H., 2005, "The Breast Cancer Book: What you need to know to Make Informed Decisions". Yale University Press Health and Wellness ISBN: 0300104138 .

[7] C. Sheng-Chih-Yang, 2005, Biomedical EngineeringApplications, Basis and Communications 17, in A Computer Aided System for Mass Detection and Classification in Digitized Mammograms, pp. 215-228.

[8] Mehul P.Sampat, 2005, "Computer-Aided Detection and Diagnosis in Mammography," Elsevier Academic Press, 2005, pp. 1195-1217.

[9] S. T. Harris Georgiou, 2007, Multi-Scaled Morphological Features for the Characterization of Mammographic Masses using Statistical Classification Schemes, in Artificial Intelligence in Medicine, pp. 267286.

[10] Stage IA, 2009, Breast cancer staging. American joint committee on cancer. $7^{\text {th }}$ edition.
[11] A. Litman, "Breaking the Breast-Cancer Stigma in Saudi Arabia," 6 May 2011.

http://msmagazine.com/blog/blog/2011/05/06/breakingthe-breast-cancer-stigma-in-saudi-arabia

[12] Ellen Warner, M.D., "Breast-Cancer Screening," The New England Journal of Medicine, 2011; 365:1025-1032

[13] Adel Aloraini, 2012, Different machine learning Algorithms for breast cancer Diagnosis. International Journal of Artificial Intelligence \& Applications (IJAIA), Vol.3, No.6, November 2012, pp: 21-30.

[14] Er. Navjot Kaur, Er. Navleen Singh Rekhi, Anand Nayyar, 2013, Review of Expert Systems based on Fuzzy logic. International Journal of Advanced Research in Computer and Communication Engineering. Vol. 2, Issue. pp 1334-1339.

[15] Lisa Rosenbaum M.D., 2014,"Invisible Risks, Emotional Choices - Mammography and Medical Decision Making," The New England Journal of Medicine; 371:1549-1552. 